\title{
Occurrence of Capping Due to Insufficient Air Escape during Tablet Compression and a Method to Prevent It
}

\author{
Tadatsugu Tanino, ${ }^{*}$ Yoshihiro Aoki, Yoshihiro Furuya, Koji Sato, Toyohiko Takeda, and \\ Taiichi MizuTA \\ Formulation Development Department of Manufacturing Division, Shionogi \& Co., Ltd., 2-1-3, Kuise-Terajima, \\ Amagasaki, Hyogo 660, Japan. Received May 15, 1995; accepted June 30, 1995
}

\begin{abstract}
A study was made on the capping of tablets containing $d$ - $\alpha$-tocopheryl acid succinate. The study determined that capping occurs due to insufficient air escape from the granules during high-speed tablet compression. To prevent this, removal of air in the granules was necessary before tableting, and thus a degassing method by compaction was studied. In consideration of the long time required for the air to escape from the granules by compaction, a slow but continuous compacting force is thought to be more effective for degassing than an instantaneous high compression force. Consequently, a system to expel the air from the lubricated granules by compaction due to the weight of the upper punch itself was established before the pre-compression roller stage, and its effect was evaluated. This system achieved efficient degassing of the granules before tableting and completely prevented the capping of tablets containing $d$ - $\alpha$-tocopheryl acid succinate at a high concentration.
\end{abstract}

Key words tablet; capping; air escape; $d$ - $\alpha$-tocopheryl acid succinate

A tablet machine used for the manufacture of drug tablets has in general a two-stage compression mechanism which consists of two rollers, a pre-compression roller and a main compression roller. Maekawa, Sakamoto et al. studied the pre-compression roller and reported that its function can effectively prevent capping by prolongation of the compression time. ${ }^{1)}$ They analyzed the ratio of the pre-compression force to the main compression force based on a theoretical equation and experimentally demonstrated that the capping frequency becomes minimum at a pre-compression ratio which makes the total compression time (sum of pre- and main compression time) the longest. This finding led to the development of several new tablet machines: a three-stage compression tablet machine, ${ }^{2)}$ a slant roller tablet machine, ${ }^{3,4)}$ and a tablet machine with an auxiliary compression system reported by Sagawa, ${ }^{5-9)}$ etc.

On the other hand, Funakoshi et al. paid renewed attention to the already known importance ${ }^{10,11)}$ of the inner die wall stress during and after compression of a tablet. As shown in Fig. 1, residual stress from the inner die wall forms a stress-concentration surface in the compressed tablet. Upward pushing of the lower punch to eject the tablet from the die additionally concentrates frictional stress on the stress-concentrated surface in the tablet. If the tablet strength is not sufficient, a part of the tablet comes off on the stress-concentrated surface, resulting in so-called capping. In order to prevent this capping phenomenon, tablet ejecting conditions were studied, and a compressive tablet ejection system was developed. In this system, the tablet is ejected while compression of the tablet is maintained with the upper and lower punches to prevent the formation of a stress-concentrated surface by the residual stress in the inner die wall. They reported that a marked cappingpreventing effect was achieved by this system. ${ }^{12,13)}$

The above two studies appear to take very different approaches. The former approach, however, can be regarded as a study aimed at the prevention of capping by prolonging the total compression time to achieve (1) relaxation of the stresses formed in the tablet along the direction of compression and inner die wall, (2) an increase in hardness of the tablet, and, at the same time, (3) relaxation of the residual stress in the inner die wall. Hence, in terms of the approach to residual stress in the inner die wall, their study is thought to have something in common with the study of Funakoshi et al. The two studies, however, differ in that the former paid attention to the compression process and the latter to the ejection process.

Thanks to the achievements of these studies, there has been much progress in tableting technology. Nevertheless, the occurrence of capping and lamination cannot be completely prevented as it also depends on the properties of the compounding ingredients, and from these we sometimes experience difficulty in industrialization. This fact suggests the presence of other unknown capping factors.

As an example of such experiences as described above, the authors studied the cause of capping and its prevention in making tablets containing $d$ - $\alpha$-tocopheryl acid succinate. ${ }^{14)}$

\section{Experimental}

1. Materials The ingredients and their weights given in Table 1 were employed as the components of lubricated granules for tableting in the present study.

2. Preparation of Lubricated Granules for Tableting These in-

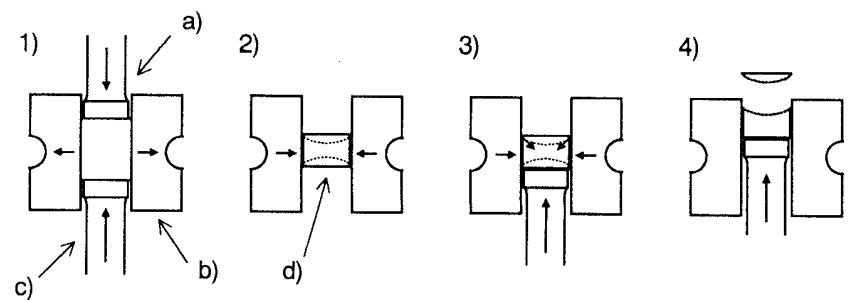

Fig. 1. Schematic Diagram of Capping Mechanism

1, Stress against die wall generated by compression; 2 , Reverse concentration of residual stress to a tablet; ( $\because .:$ : Streee-concentrated portion) 3 , Additional concentration of frictional stress by ejection; 4, Capping occurrence; a) Upper punch; b) die; c) lower punch; d) compressed tablet.

(C) 1995 Pharmaceutical Society of Japan 
Table 1. Tablet Formula

\begin{tabular}{lclll}
\hline \multicolumn{1}{c}{ Ingredients } & Weight $(\mathrm{mg})$ & Grade & \multicolumn{1}{c}{ Bulk suplier } & \\
\hline Dicethiamine hydrochloride & 50 & JPC ${ }^{a)}$ & Shionogi \& Co., Ltd. & Osaka (Japan) \\
Riboflavin & 5 & JP12 & Tokyo Tanabe Co., Ltd. & Tokyo (Japan) \\
Pyridoxine hydrochloride & 25 & JP12 & Alpus Pharmaceutical Ind. Co., Ltd. & Gifu (Japan) \\
d- $\alpha$-Tocopheryl acid succinate & 50 & USP23 & Eastman Chemical Company & Tennessee (U.S.A.) \\
Hydroxypropylcellulose SL & 4 & JP12 & Nippon Soda Co., Ltd. & Tokyo (Japan) \\
Lactose & 46 & JP12 & D.M.V. International & Veghel (Netherlands) \\
Corn starch & 18 & JP12 & Japan Maize Products Co., Ltd. & Tokyo (Japan) \\
Crystalline cellulose & 18 & JP12 & Asahi Chemical Ind. Co., Ltd. & Tokyo (Japan) \\
Magnesium stearate & 4 & JP12 & Taihei Chemical Ind. Co., Ltd. & Osaka (Japan) \\
Total & 220 & & & \\
\end{tabular}

a) Japanese Pharmaceutical Codex (1993).

Table 2. Manufacturing Equipment

\begin{tabular}{llll}
\hline Process & Equipment & Type & Manufacturer \\
\hline Dry mixing & High speed mixer & FS, GS, 5JD & Fukae Powtec \\
Agitating granulation & High speed mixer & FS, GS, 5JD & Fukae Powtec \\
Drying & Fluidized bed granulator & WSG-5 & Glatt GmbH \\
Sizing & Power mill & P3 & Dalton \\
Final mixing & Twin shell blender & Y5 1 & Yamada Iron Works \\
Lubrication & Twin shell blender & Y51 & Kamada Iron Works \\
Compression & Tablet machine & RTM-S30K-2S-T30 & Kikushu, Shionogi \\
& & RTM-S30K-2S-T30-2 & (Conventional type) \\
& & (Equipped with a degassing mechanism)
\end{tabular}

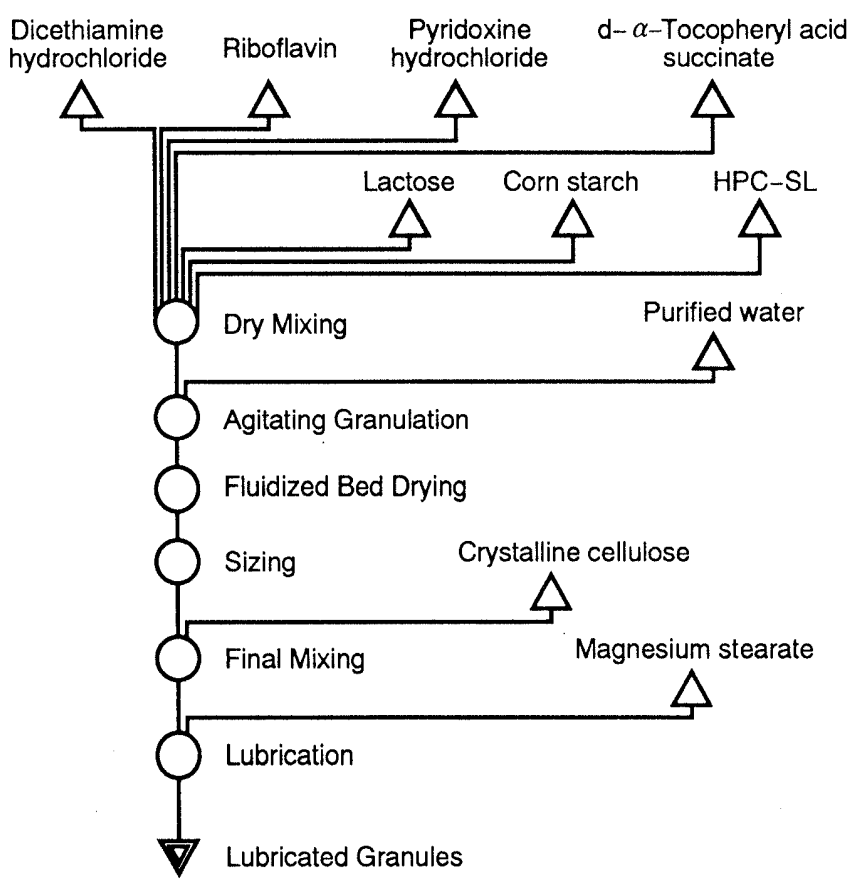

Fig. 2. Production Flow Chart of Lubricated Granules

gredients, from dicethiamine hydrochloride to corn starch, were granulated by the wet agitating granulation method. After the drying and sizing operation, the remaining ingredients were added to the sized granules and then mixed and lubricated. Figure 2 shows the preparation flow chart, while Table 2 compiles the manufacturing equipment used.

3. Tableting Experiment 1) Static Tableting Experiment: Using a static compression testing machine (a product of Tokyo Koki), $220 \mathrm{mg}$ of lubricated granules were compressed into tablets with various thicknesses at a compression speed of $0.1 \mathrm{~cm} / \mathrm{min}$, and the hardness and the presence or absence of capping in the tablet obtained were examined.

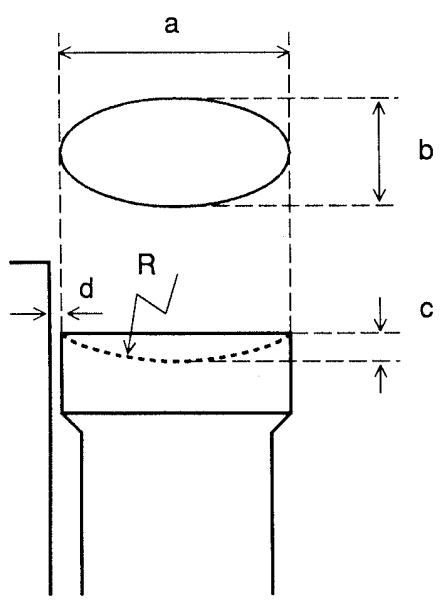

Fig. 3. Punch Shape and Size

a, $12.0 \mathrm{~mm}$; b, $6.5 \mathrm{~mm} ; \mathrm{c}, 1.25 \mathrm{~mm}$;, $0.02-0.05 \mathrm{~mm}$. R $=15 \mathrm{~mm}$.

The elliptic die and punch depicted in Fig. 3 were used.

2) Dynamic Tableting Experiment: Using a slant-roller type two-stage compression tablet machine (RTM-S30K-2S-T30; Kikusui Seisakusho) which has 30 tableting stations, the compression ratio of the pre- to main compression force was varied to obtain an optimum ratio. At two settings of rotation speed of the tablet machine, 30 and $40 \mathrm{rpm}$, a study was made of whether the frequency of capping and lamination is minimized at a pre-compression ratio of $50 \%$ as reported by Maekawa et al. The same punch and die as used for the static tableting experiment were used (Fig. 3), and an open feedshoe was used for feeding of the lubricated granules.

3) Long Non-stop Experiment: Employing the optimum pre- to main compression force ratio obtained in the dynamic tableting experiment, the tablet machine was run continuously at rotation speeds of 30 and $40 \mathrm{rpm}$ for a long time, and changes in the frequency of capping were studied with the passage of time.

4) Measurement of Capping Frequency: 50 tablets were placed in a 
friabilator, impact was applied to the tablets for $10 \mathrm{~min}$, and then the number of tablets which underwent capping was counted. The mean from 5 tests was used as the capping frequency.

5) Measurement of Tablet Hardness and Thickness: Tablet hardness was measured using an Erweka hardness tester (TBH-28), and the mean of 10 tablets was calculated. Tablet thickness was measured using a thickness gauge, and the mean was also calculated using 10 measurements.

4. Experiment on Prevention of Capping The above-described experiments, from 3-2) through 3-5), were conducted using a modified tablet machine (RTM-S30K-2S-T30-2) with the ability to expel the air in the lubricated granules in a die by the compacting weight of the upper punch itself, and the machine's effect on preventing capping was evaluated.

\section{Results and Discussion}

1. Static Tableting Experiment As depicted in Fig. 4, an inverse relation was found between tablet thickness and tablet hardness. As designed thickness, $4.40 \mathrm{~mm}$ was selected, which gives a sufficient hardness of $10 \mathrm{~kg}$ or more for the handling of tablets. At each thickness tested, however, no capping was observed, indicating good compressibility of the lubricated granules prepared in this experiment.

2. Dynamic Tableting Experiment 1) Relationship between Tablet Thickness for Pre-compression and Capping Frequency: Figure 5 shows the relationship between tablet thickness and capping frequency when only

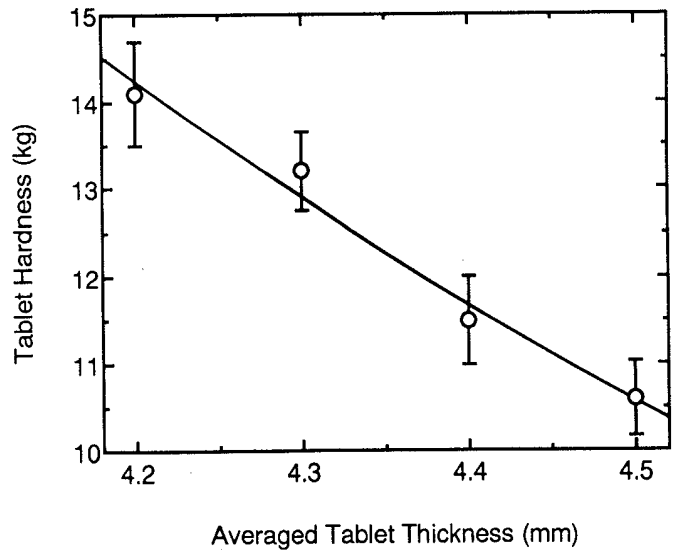

Fig. 4. Relationship between Averaged Tablet Thickness and Tablet Hardness

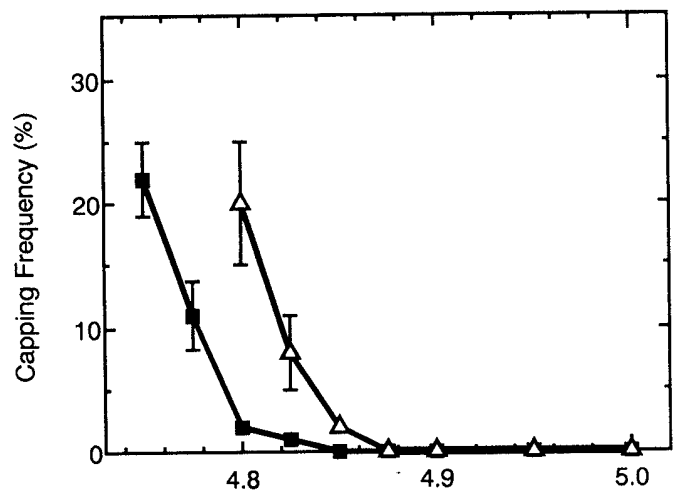

Averaged Tablet Thickness for Pre-compression (mm)

Fig. 5. Relationship between Averaged Tablet Thickness for Precompression and Capping Frequency without Main Compression

Rotation speed: $-\square-30 \mathrm{rpm} ;-\triangle-40 \mathrm{rpm}$ the pre-roller was used for compression tableting without using the main roller.

At the rotation speed of $30 \mathrm{rpm}$, capping was observed at tablet thicknesses of $4.85 \mathrm{~mm}$ and less, and the capping frequency increased when the pre-compression force was increased. At the rotation speed of $40 \mathrm{rpm}$, on the other hand, capping occurred at tablet thicknesses of $4.88 \mathrm{~mm}$ and less, indicating the occurrence of capping at a smaller pre-compression force than that in the case of $30 \mathrm{rpm}$.

2) Relationship between Tablet Thickness for Pre- and Main Compression and Capping Frequency: As shown in Fig. $6 \mathrm{a}$, at the rotation speed of $30 \mathrm{rpm}$ and a tablet thickness for the main compression of $4.30 \mathrm{~mm}$, capping occurred at all tablet thicknesses during the precompression. However, when the thickness for the main compression was set at $4.40 \mathrm{~mm}$, the designed value, capping could be prevented within a pre-compression thickness range of 4.85 to $4.98 \mathrm{~mm}$. When the main compression thickness was set at $4.50 \mathrm{~mm}$, the range causing no capping became even broader. At the rotation speed of $40 \mathrm{rpm}$ and a main compression thickness of $4.40 \mathrm{~mm}$, as shown in Fig. $6 \mathrm{~b}$, the range of the precompression thickness causing no capping became narrower compared with that at $30 \mathrm{rpm}$.

In the dynamic tableting experiment, as described above,

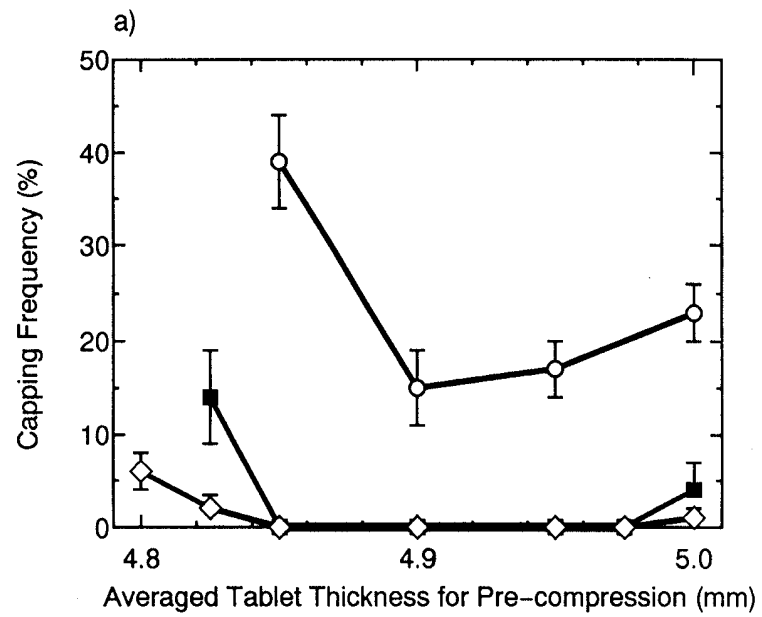

b)

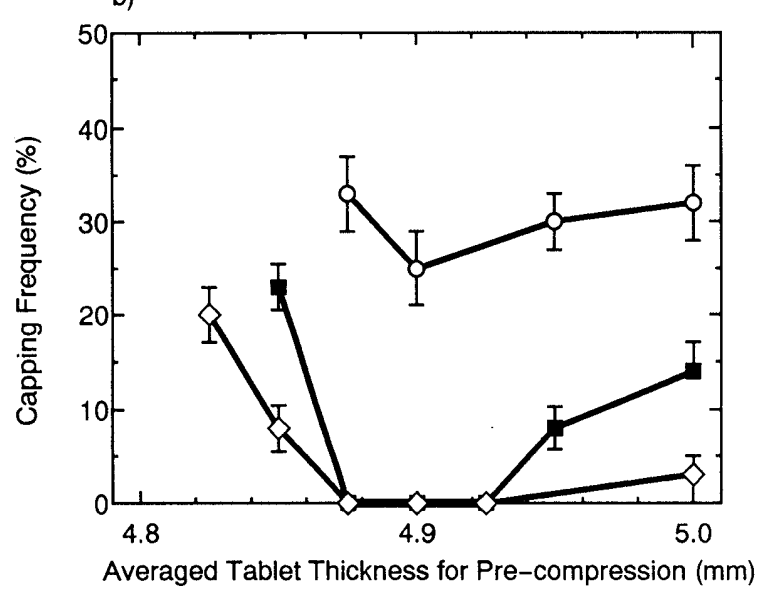

Fig. 6. Relationship between Averaged Tablet Thickness for Pre- and Main Compression and Capping Frequency

Tablet thickness for main compression: $-\mathrm{O}-4.30 \mathrm{~mm} ;-\square-4.40 \mathrm{~mm} ;-\diamond-$ $4.50 \mathrm{~mm}$; Rotation speed: a) $30 \mathrm{rpm}$, b) $40 \mathrm{rpm}$. 
the capping frequency changed greatly depending on the tablet thicknesses during pre- and main compression; these results differed greatly from those obtained in the static tableting experiment, in which no capping occurred.

3) Experiment of Long Continuous Run of Tablet Machine: In this experiment, the tablet thickness for the main compression was set at the designed thickness of $4.40 \mathrm{~mm}$, and the tablet machine was run for a long time at a pre-compression tablet thickness of $4.90 \mathrm{~mm}$, which is thought from Fig. 6 to be least likely to undergo capping. The frequency of capping was measured with the passage of time. As depicted in Fig. 7, tablets could be produced stably for some time, but the capping phenomenon suddenly appeared at a certain point in time, and it continued thereafter. The time the capping appeared was earlier at the rotation speed of $40 \mathrm{rpm}$ than that of $30 \mathrm{rpm}$.

4) Assessment of Optimum Pre-compression Ratio: In the present study, the pre-compression ratio was calculated using Eq. 1, while as the initial tablet thickness, the thickness at which stress begins to appear for the first time in the gradual compression of the lubricated granules, was employed in accordance with the method of Maekawa et al. ${ }^{1)}$

$$
r(\%)=100(i-p) /(i-m)
$$

where, $r$ : pre-compression ratio, $i$ : initial tablet thickness, $p$ : thickness for pre-compression, $m$ : thickness for main compression.

The initial lubricated granule thickness employed in the present study is $5.15 \mathrm{~mm}$. Thus, based on the results shown in Fig. 6, the pre-compression ratio that reduces capping to the smallest frequency can be calculated to be approximately $33 \%$, where the pre- and main tablet thickness are 4.90 and $4.40 \mathrm{~mm}$, respectively. This differs considerably from the report by Maekawa et al. that the capping ratio becomes the smallest at a pre-compression ratio of $50 \%$. Meanwhile, Funakoshi et al. reported that the capping frequency becomes small at a pre-compression ratio of $80 \%$ or higher. ${ }^{12)}$ The difference between those two groups, however, is due to a difference in their interpretation of the initial thickness. It is thought that the same result would be obtained using matching criterion.

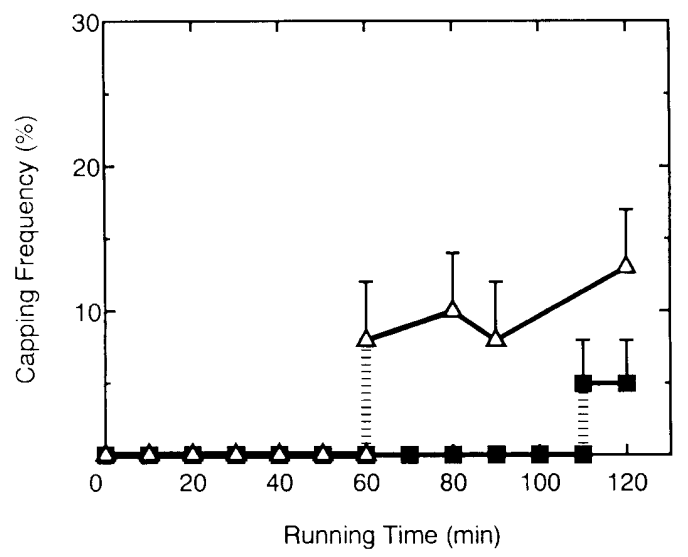

Fig. 7. Change in Capping Frequency during Running of Tablet Machine

Rotation speed: $-\mathbf{0}-30 \mathrm{rpm}: \cdots \triangle-40 \mathrm{rpm}$.
The above dynamic tableting experiment revealed the following two facts:

(1) The pre-compression ratio minimizing the capping frequency is considerably smaller for the tablet composition employed in the present study than the previous general ratio.

(2) The occurrence of capping is dependent on the running time of the tablet machine.

These two findings cannot be fully explained by the mechanical hypothesis of Funakoshi et al. alone, in which the cause of capping is attributed to friction generated at the time of ejection of the tablet by residual stress in the inner die wall. The results of the present study suggest the presence of some special cause.

3. Investigation into Cause of Capping The changes in the punch and die of the tablet machine which occurred when capping began to appear when the machine was run for a long time were observed. As shown in Fig. 8, the tablet granules had firmly adhered around the lower punch where there were no such granules initially. It was found that the granules contaminating the punch were blocking

a)

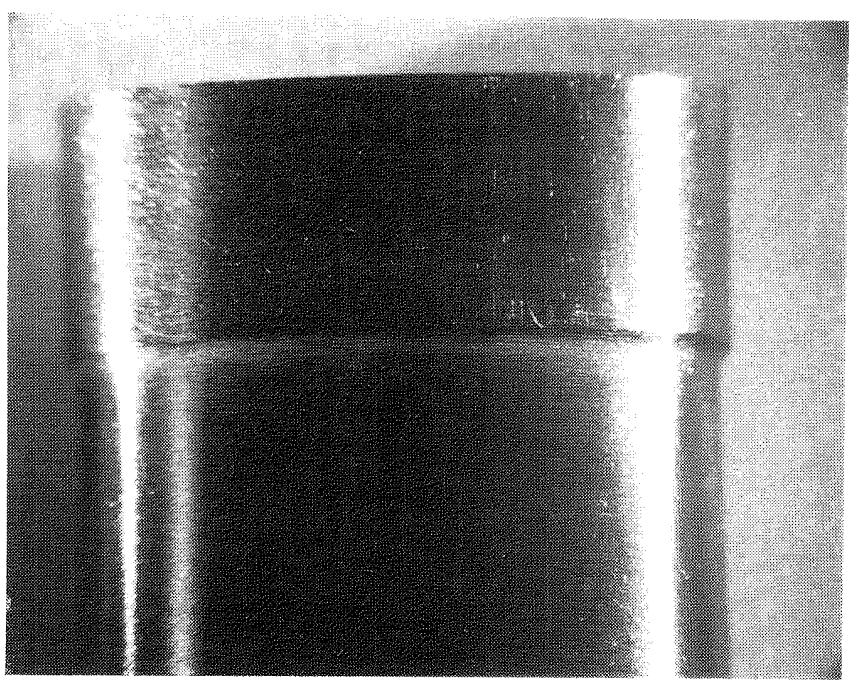

b)

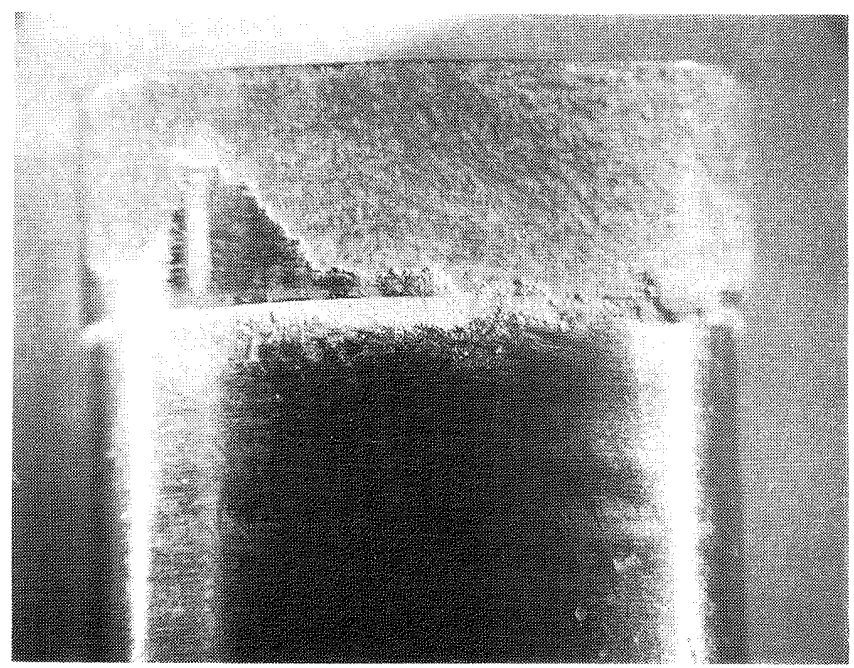

Fig. 8. Change in Appearance of Lower Punch during Tableting a) Initial, b) at the time when tablets began to cap. 
the gap between the die and punch so that air contained in the lubricated granules could not escape from the die when they were compressed.

Figure 9 shows the external appearance of a tablet undergoing capping in this experiment. The tablet was split in two halves at the center. This indicates that this mechanism of capping differs from the usual, in which a fragment of the tablet is detached from the upper surface of the tablet.

On the basis of the results of the above experiments and observation, the following can be said with regard to capping observed in the present study.

(1) The tablet formula employed in the present study includes $d$ - $\alpha$-tocopheryl acid succinate, an oily, adhesive substance with a low melting point, at a rate of about $23 \%$, and thus the granules are likely to undergo plastic deformation. Accordingly, the inter-granular spaces, through which air normally escapes, are likely to be obstructed, and instantaneous application of a precompression force using the compressive roller at a high compression ratio causes insufficient degassing so that much air remains entrapped within the granules, resulting

a)

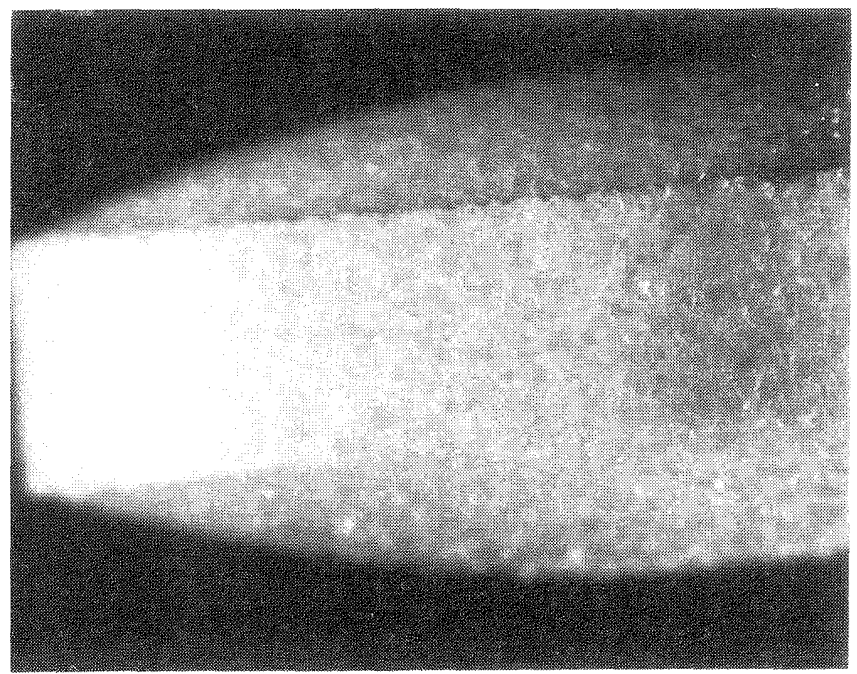

b)

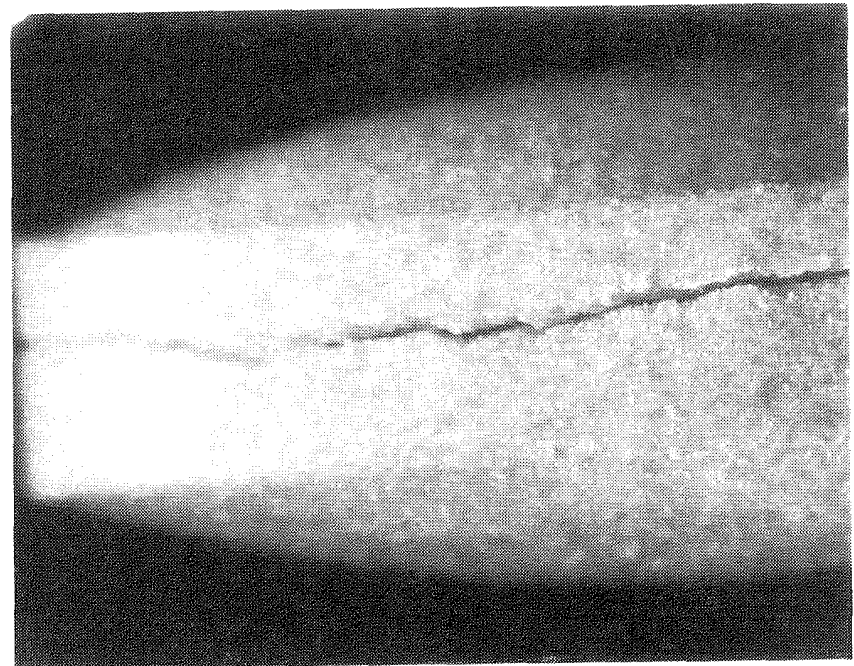

Fig. 9. The Appearance of Normal (a) and Capped Tablets (b) in capping.

(2) A pre-compression ratio of $33 \%$, which exhibited the lowest capping frequency in the dynamic tableting experiment, is considerably smaller than the usual ratio, but the degassing efficiency is best at that ratio.

(3) In the long operation of the tablet machine, capping appeared suddenly at a certain point in time. This occurs as follows: Granules entrapped in the gap between the punch and die are softened due to the heat generated by the friction of the punch and die; they gradually attach to the wall of the punch and die and decrease the air escape efficiency by blocking the gap between the punch and die. In other words, capping appears suddenly when the air escape efficiency has decreased to a point where it cannot meet the compression speed. This theory adequately explains the fact that capping appeared earlier at a rotation speed of $40 \mathrm{rpm}$, which generates more heat in the punch and die and a higher compression speed than at $30 \mathrm{rpm}$.

This theory is also supported by the fact that even after the appearance of capping, tableting was achieved again without capping for a certain period of time by suspending the system to cool down the punch and die, and by removing the adhered substances.

4. Modification of Tablet Machine for Prevention of Capping The above experiments elucidated that the poor escape of intra-granular air in association with high-speed compression is the major cause of capping observed in the tableting of granules containing $d$ - $\alpha$-tocopheryl acid succinate at a high concentration. This capping phenomenon arising from the loss of an escape route of intragranular air at the time of compression has already been reported. ${ }^{15)}$ However, there have been few reports that present actual examples as given in the present study. In addition, most approaches to the prevention of capping have been negative methods, such as reduction of the rotation speed of the tablet machine.

Therefore, with the objective of preventing capping of this type, the authors studied a method which can efficiently remove the air entrapped in the lubricated granules.

The air entrapped in the lubricated granules filled in a die is thought to escape from the system upon compression according to the Eq. 2.

$$
-d x / d t=k(p, T)\left[P(t, p)-P_{0}\right]
$$

where, $x$ :amount of air entrapped in the granules,

$p$ :compression force,

$T$ :tablet machine running time,

$t$ :compression time, $k(p, T)$ : degassing efficiency,

$P(t, p)$ :pressure of air in the granules under compression force $p$,

$P_{0}$ :environmental air pressure

From Eq. 2, the following three approaches can be thought as efficient methods to remove air in the granules:

(1) Through stabilization of the value of $k(p, T)$, maintaining the initial level of tableting.

In this approach, heat generation by long duration running of the tablet machine is prevented, thereby preventing the punch-die gap from being blocked as a result of softening and adhesion of the granules. As a basic 
method, cooling of the punch and die is considered.

(2) Making the difference between intra-granule air pressure $P(t, p)$ and atmospheric pressure $P_{0}$ at the time of compression as large as possible.

To achieve this, one method is to increase pre-compression force $p$. However, because a pre-compression ratio of $33 \%$ was found to be the best in the dynamic tableting experiment, it is thought to be more effective to reduce environmental air pressure, $P_{0}$, rather than increasing $p$. Practically, this approach requires vacuum tableting or tableting with a reduced pressure system.

(3) Prolonging compression time $t$ to facilitate air escape.

This approach requires the establishment of a system which assures enough time to permit the escape of a sufficient amount of air entrapped in the granules.

Of the three above methods, the approach described under (3) was investigated in the present study.

First, before assuring compression time, the compression force for degassing was studied. For this objective, the relationship between the compaction pressure for the granules employed in the present study and the apparent

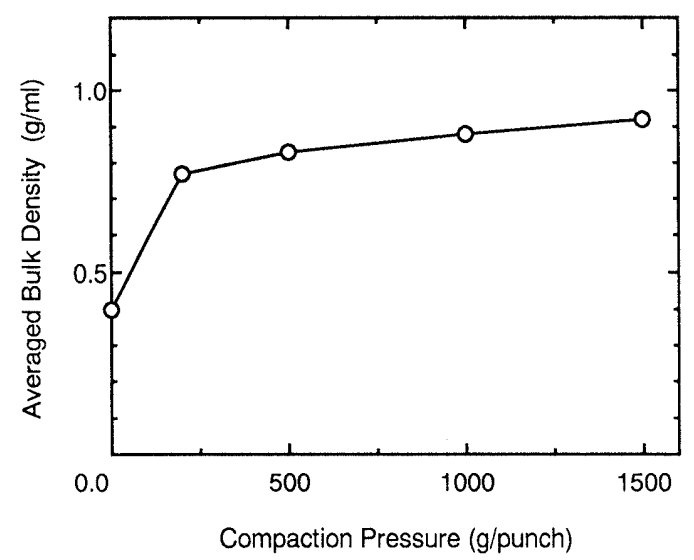

Fig. 10. Change in Averaged Bulk Density as a Function of Compaction Pressure

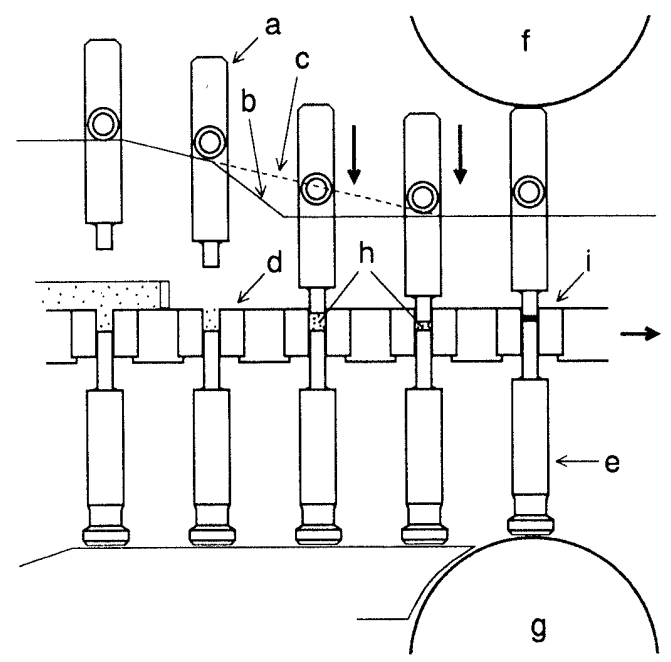

Fig. 11. Schematic Diagram of the Degassing Mechanism before Pre-compression

a, Upper punch; $b$, modified upper punch guide rail; c, conventional upper punch guide rail; $\mathrm{d}$, die; e, lower punch; $\mathrm{f}$, upper pre-compression roller; $\mathrm{g}$, lower pre-compression roller; $h$, granules under degasification by upper punch weight; $i$ pre-compression. bulk density was analyzed using the equipment employed in the previous report. ${ }^{16)}$ As depicted in Fig. 10, the apparent bulk density increased rapidly up to a per-punch compaction pressure of $500 \mathrm{~g}$, indicating degassing, but above this pressure, the increase in the apparent bulk density, which is attributed to degassing, became slow. Thus, a compaction pressure of 500 to $1000 \mathrm{~g}$ /punch was decided upon as the degassing pressure. Since this selected pressure range is nearly the same as the weight of the upper punch, a method was studied in which the granules are compacted and degassed by a free fall of the upper punch utilizing its weight. This system is diagrammatically presented in Fig. 11. In this system, unlike instantaneous compression as designed for the roller compression, a comparatively long compacting/degassing time can be obtained, starting from the time of completion of granule weight adjustment to pre-compression with the pre-roller.

In order to provide the tablet machine with this mechanism, as depicted in Fig. 12, the weight of the upper punch was increased to $800 \mathrm{~g}$, which is about 1.5 times heavier than a conventional punch, and the course of the upper punch guide rail was modified. ${ }^{17)}$

5. Experimental Tableting Using the Modified Tablet Machine 1) Relationship between Pre-compression Thickness and Capping Frequency: Figure 13 shows the (a)

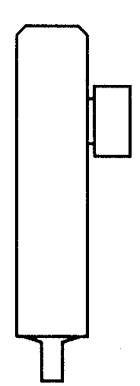

(b)

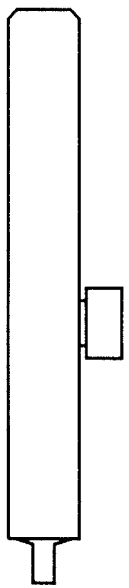

Fig. 12. Appearance of Conventional (a) and Weighted Upper Punch (b)

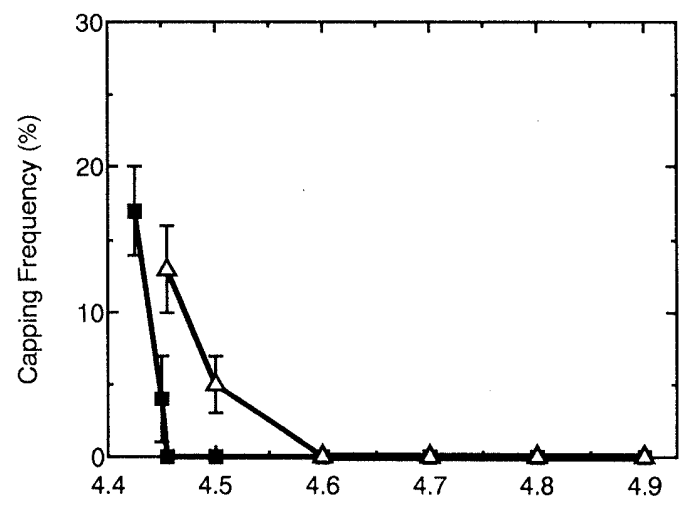

Averaged Tablet Thickness for Pre-compression ( $\mathrm{mm}$ )

Fig. 13. Relationship between Averaged Tablet Thickness for Precompression and Capping Frequency when the Degassing Mechanism was Used without Main Compression

Rotation speed: $-\mathbf{n}-30 \mathrm{rpm} ;--\triangle-40 \mathrm{rpm}$. 
relationship between tablet thickness and capping frequency when only the pre-roller was used for compression, but not the main roller. At both rotation speeds of 30 and $40 \mathrm{rpm}$, pre-compression shaping was achieved up to a tablet thickness of $4.60 \mathrm{~mm}$ without capping, enabling the application of a much higher pre-compression force compared with Fig. 5, in which no degassing system was employed. This indicates that a considerable amount of air has been removed prior to pre-compression by the degassing mechanism shown in Fig. 11. As a result of the installation of this system, it became possible to apply a pre-compression ratio of $50 \%$ (pre-compression tablet thickness: $4.78 \mathrm{~mm}$ ) which can minimize the frequency of usual capping as shown in Fig. 1.

2) Relationship Between Pre- and Main Compression

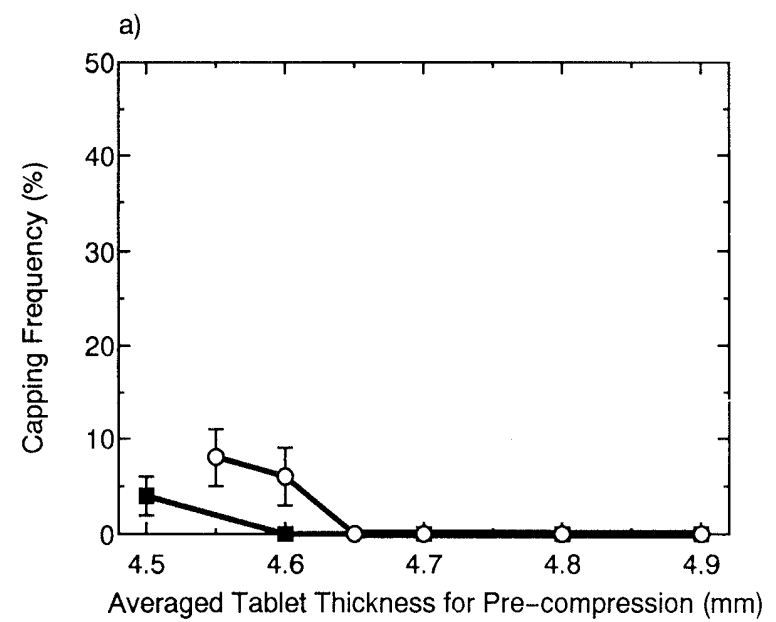

b)

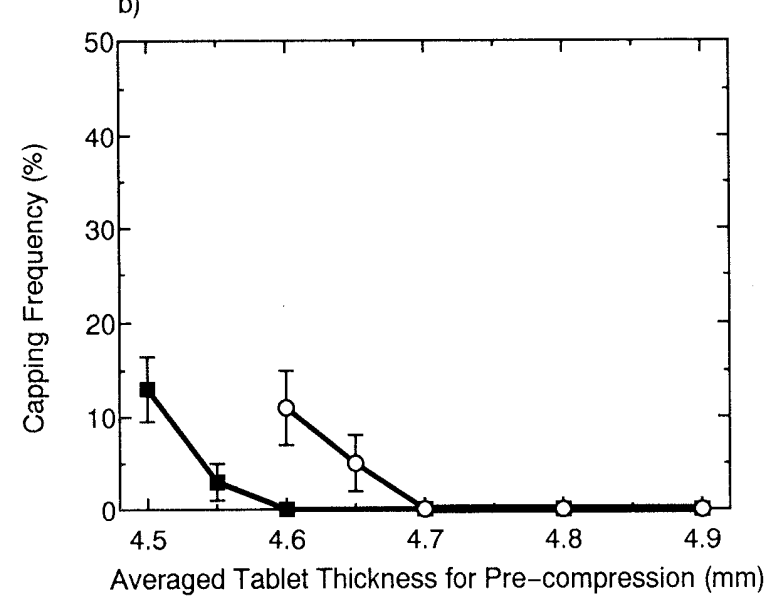

Fig. 14. Relationship between Averaged Tablet Thickness for Pre- and Main Compression and Capping Frequency when the Degrassing Mechanism was Used

Tablet thickness for main compression: $-\mathrm{O}-4.30 \mathrm{~mm}$; $-\mathbf{\square}-4.40 \mathrm{~mm}$; Rotation speed: a) $30 \mathrm{rpm}$; b) $40 \mathrm{rpm}$.

Table 3. Tablet Hardness

\begin{tabular}{ccc}
\hline Tablet machine & $\begin{array}{c}\text { Pre-compression } \\
\text { ratio (\%) }\end{array}$ & $\begin{array}{c}\text { Tablet hardness }(\mathrm{kg}) \\
(\bar{X}, n=10)\end{array}$ \\
\hline $\begin{array}{c}\text { Conventional type } \\
\text { (T30) }\end{array}$ & 33 & 9.6 \\
$\begin{array}{c}\text { Equipped with a degassing } \\
\text { mechanism (T30-2) }\end{array}$ & 50 & 11.8 \\
\hline
\end{tabular}

Tablet Thicknesses and Capping Frequency: At both rotation speeds of 30 and $40 \mathrm{rpm}$, when the main compression tablet thickness was set at the designed value of $4.40 \mathrm{~mm}$, the range of capping-free pre-compression tablet thickness, as depicted in Figs. 14a and 14b, became greatly wider compared with Fig. 6. In addition, even at a main compression tablet thickness of $4.30 \mathrm{~mm}$, a capping-free pre-compression tablet thickness range appeared. These results confirmed that the degassing system can greatly improve the tablet machine's compression shaping ability and effectively prevent capping occurring from poor degassing.

3) Experiment of Long and Continuous Running of Tablet Machine Equipped with Degassing System: In an 8-h continuous operation employing a pre-compression tablet thickness of $4.78 \mathrm{~mm}$ and a main compression tablet thickness of $4.40 \mathrm{~mm}$, no sudden occurrence of capping was observed at rotation speeds of either 30 nor $40 \mathrm{rpm}$ of the tablet machine. This confirmed that the system is capable of removing enough air to prevent capping, even when the gap between the punch and die is blocked to some degree by adhesion of the granules.

Our degassing system made it possible to stably produce tablets containing $d-\alpha$-tocopheryl acid succinate at a high concentration, the formula employed in the present study. At the same time, because it allowed a pre-compression ratio of $50 \%$, and a pre-compression thickness of $4.78 \mathrm{~mm}$, the tablet hardness increased, as shown in Table 3, by about $20 \%$ compared with that obtained from the conventional tablet machine.

\section{Conclusion}

Throughout this investigation, the following information was obtained.

(1) The capping phenomenon observed in the tableting of a formula containing $d$ - $\alpha$-tocopheryl acid succinate at a high concentration is mainly caused by obstruction of the escape route for intra-granular air under high-speed compression.

(2) For removal of the air entrapped in the lubricated granules, low-pressure, long-time compacting is more effective than high-pressure, instantaneous compression with the pre-compression roller.

(3) A degassing system utilizing the weight of the upper punch as the compressive force for degassing was developed and confirmed to achieve good prevention of capping. A three-stage compression comprised of degassing compaction, pre-compression and main compression is effective for producing tablets from the formula employed in this study.

Acknowledgment We thank Dr. Teruo Sakamoto, a former consultant of Shionogi Pharmaceutical Co., Ltd., and Mr. Izumi Saito, Mr. Kazumasa Kumai, research staff members of the developmental research laboratories of the same company, for their valuable advice. Special thanks are also due to Ms. Sadami Tanami, a research staff member of the manufacturing division of the same company, for her excellent assistance.

\section{References}

1) Maekawa H., Sakamoto T., Masui Y., Imagawa H., Yakuzaigaku, 29, 90-94 (1969).

2) Maekawa H., Sakamoto T., Imagawa H., Yakuzaigaku, 29, 97-102 
(1969).

3) Maekawa H., Sakamoto T., Imagawa H., Yakuzaigaku, 29, 102-107 (1969).

4) Maekawa H., Sakamoto T., Yakuzaigaku, 32, 124-130 (1972).

5) Sagawa Y., Sakamoto T., Maekawa H., Yakuzaigaku, 36, 135-144 (1976).

6) Sagawa Y., Sakamoto T., Maekawa H., Yakuzaigaku, 36, 145-151 (1976).

7) Sagawa Y., Sakamoto T., Maekawa H., Yakuzaigaku, 36, 189-193 (1976).

8) Sagawa Y., Sakamoto T., Maekawa H., Yakuzaigaku, 37, 15-22 (1977).

9) Sagawa Y., Sakamoto T., Maekawa H., Yakuzaigaku, 37, 80-87 (1977).
10) Windheuser J. J., Misra J., Eriksen S. P., Higuchi T., J. Pharm. Sci., 52, 767-772 (1963).

11) Higuchi T., Shimamoto T., Eriksen S. P., Yashiki T., J. Pharm. Sci., 54, 111-118 (1965).

12) Funakoshi Y., Kajiura T., Asogawa T., Zairyo, 18, 574-553 (1969).

13) Funakoshi Y., Asogawa T., Maeda T., Zairyo, 25, 671-674(1976).

14) Tanino T., Furuya Y., Sato K., Takeda T., Inazu K., Abstracts of Papers, The 111th Annual Meeting of Pharmaceutical Society of Japan, Tokyo. March 1991, Part 4, p. 98.

15) Burlinson H., J. Pharm. Pharmacol., 6, 1055-1066 (1954).

16) Tanino T., Aoki Y., Sato K., Takeda T., Mizuta T., Yakuzaigaku, 54, 238-245 (1994).

17) Shionogi \& Co., Ltd., Japan. Patent Hei-4-111997 (1992). 